\title{
Review on Bitcoin Price Prediction Using Machine Learning and Statistical Methods
}

\author{
I.Sibel Kervanc1 ${ }^{1 *}$, (D) M.Fatih Akay ${ }^{2}$ \\ ${ }^{1}$ Corresponding Author; Gaziantep Üniversitesi Bilgi İşlem Daire Başkanlığı; skervanci@gantep.edu.tr; \\ $+903423171978$ \\ ${ }^{2}$ Çukurova University, Faculty of Engineering, Department of Computer Engineering; mfakay@cu.edu.tr
}

Received 27 July 2020; Revised 25 Nov 2010; Accepted 2 December 2020; Published online 30 December 2020

\begin{abstract}
Bitcoin is invented in 2009 by the pseudonymous Satoshi Nakamoto. Bitcoin is a decentralized digital currency system [1]. Bitcoin is the most acknowledged cryptocurrency in the world, which provide it interesting for financier. The cryptocurrency market capitalization on date 22nd July 2020 value represents roughly USD 277 billion of dollars, bitcoin representing $62 \%$ of it. However, a disadvantage for investors is the difficulty of predicting the price of bitcoin due to the high volatility of the bitcoin exchange rate. Measurement, estimation, and modeling of currency exchange rate volatility compose a significant research area. For this reason, a lot of studies done about bitcoin price prediction both Machine Learning (ML) and Statistical Methods. In comparison studies, ML methods perform better in general. This review is a comprehensive study on how we can better predict bitcoin prices by grouping previously done studies. The presentation of Bitcoin price prediction studies in groups reveals, the difference from other review studies. These are statistical methods, ML and statistical methods, ML-ML, frequency effect of selected time, effect of social media and web search engine, causality, optimization of hyperparameters methods.
\end{abstract}

Keywords: Bitcoin price prediction, machine learning, statistical methods.

\section{Introduction}

Virtual currency is a relatively new phenomenon in global finance. Therefore, its identity, structure, and function constantly improve; however, in the meantime, it is increasingly recognized as a better medium for global finance with great potential [2]. Bitcoin was developed to avoid using reliable third parties such as banks, cards, and governments, as well as to avoid time delays and money redirection costs [3]. Bitcoin, one of the virtual currencies, is distinguished from the others by its enormous potential. The majority of cryptocurrencies are largely clones of bitcoin or other cryptocurrencies [4]. For this reason, it attracted the attention of many researchers, and many articles on price prediction were published by using both statistical and ML methods [5]-[12]. Statistics is a collection of tools improved over hundreds of years for summarizing the dataset and quantifying properties of an area such as an instance of observations. Additionally, Statistical Methods constitute a significant base area of mathematics, which is required for reaching a deeper understanding of the behavior of ML algorithms. MLsearches for patterns in the dataset and tries to conclude, just as people would, while statistical methods works within the process of obtaining some meaningful information by analyzing the dataset correctly.

There are many different options to create a time series dataset for Bitcoin [13]-[16].The bitcoin dataset can obtain a rather granular time interval in theory. Thus, it is possible to obtain many different datasets by considering different time intervals. In addition to time variability, bitcoin is an environment where anyone can see transfers. Among the features existing in the blockchain structure such as volume, researchers can add features that were established with the causality relationship to the dataset.

Apart from the features added from the blockchain, they can also add extra features that are believed to have a causality relationship or that are believed to affect bitcoin price, such as dollar rate, gold price rate, social media, one or more of the other cryptocurrencies and so on [17]-[27]. For these reasons, while examining the studies on bitcoin price estimation, it is necessary to examine the results due to the differences in methods, the datasets, as well as investigating the effects of causality relationships on the results. A study that added extra features may perform better with one of the statistical methods while 
two studies using the same approach may obtain a better result by conducting hyperparameter optimization better.

The most commonly used statistical and ML algorithms are as follows. Examples of Statistical methods include moving average (MA), a simple moving average (SMA), auto-regressive integrated moving average (ARIMA), the generalised autoregressive conditional heteroskedasticity (GARCH), autoregressive distributed lag (ARDL) and nonlinear autoregressive distributed lag (NARDL) [7], [8], [9]. Additionally, ML methods include support vector machine (SVM), multi-layer perceptron (MLP), linear regression (LR), recurrent neural network (RNN), convolutional neural network (CNN), generalized regression neural networks (GRNN), long-short term memory (LSTM) and gated recurrent unit (GRU) [13], [28]-[31].

Statistics is defined as the study of collection, analysis, interpretation, forecasting, presentation, and organization of dataset while ML is effectively used in various fields such as forecasting [32], real-time advertisement, malvare detection [33], and text-based sentiment analysis [34]. Both are used in forecasting problems. In the comparative studies of ML and statistical methods, ML methods perform better in general [5], [6], [10]-[12].

Based on reference [35], it was determined that there is no seasonal relationship in the Bitcoin dataset, which is a disadvantage for statistical methods. This is also shown as the reason for ML models to provide better performance results.

Based on reference [32], it was discovered that especially deep learning methods are more successful than statistical and classical ML methods in datasets containing nonlinear and hidden patterns, such as the bitcoin dataset. There are also hybrid models consisting of statistical and ML algorithms working together. The references [5] and [6] are studies that can be examples of this model.

In this report, the studies on Bitcoin Price prediction were examined. The studies were presented in detail in the "Literature Review" section.

Then, in the "Result \& Discussion"section, the studies were visualized according to the causality relationship, the results of the methods, etc. The main contribution of the article is that following the examination of the bitcoin price prediction, they were drawn into groups and analyzed in detail, which enabled drawing conclusions from the articles according to the groups. For those who want to conduct researches about Bitcoin price, this study may prove vital thanks to the results that were summarized for all the groups.

\section{Literature Review}

In this report, we used articles published on bitcoin price prediction to compare ML and statistical methods. The articles chosen were among those published in recent years, while older articles were also included because some methods lost their popularity in recent times. The articles were analyzed in terms of the frequency of the dataset, the causality relationship, and the method.

\subsection{Statistical Methods}

Based on reference [7], ARIMA, AR, and MA were conducted in the time series dataset. The result of this paper, using the ARIMA model on the Bitcoin price is predicted with an accuracy of $90.31 \%$. Thus, it can be stated that the best result was attained by using ARIMA.

Complex processes cannot be represented and successful results cannot be achieved using the linear model in the real world. However, the ARIMA model has one advantage. It has specific components that define trend, error, and seasonality separately (p, d, q). Therefore, nonlinear models can be represented and can yield good results as in reference [7].

Based on reference [8], only the ARIMA algorithm was used to predict the Bitcoin prices. To find the lowest MSE, the researchers used fit function with different values in the ARIMA algorithm and found 
the lowest as MSE $=170962.195$. This result differs from other studies because of refraining from using a scale function.

Based on reference [9], some type of GARCH models were used for daily closing prices between July 18, 2010, and October 1, 2016. As a result, the researchers discovered that AR-CGARCH was the best model.

\subsection{Machine Learning -Statistical Methods}

Based on reference [10], four methods were used for price predictions, which included Logistic regression, Support vector machine, RNN, and ARIMA. In terms of prediction accuracies for these four methods, ARIMA has $53 \%$ for only next day price prediction while performing poorly for longer terms such as using price prediction of the last few days for prices of next 5-7 days. RNN performs consistently up to 6 days such as 50\%. The logistic regression-based model's assumptions were not violated; it can only conduct classifications accurately if a separable hyperplane exists with $47 \%$ accuracy. SVM has an accuracy of $48 \%$.

Based on reference [5], the researchers compared the input selection of ARIMA and VAR while using Support Vector Regression (SVR) and MLP network, which is a process called the Hybrid Model. Trials indicate that the SVR method surpassed the MLP networks for each entry. In addition, hybrid methods perform better than naive ARIMA and VAR.

Based on reference [6], the researchers used MLP Based Nonlinear Auto-Regressive Network with eXogenous (MLP-based NARX) and MA as technical indicators for the prediction model. The researchers also used an optimizer called The Particle Swarm Optimization (PSO) algorithm. This model's results were acceptable in general while the correlation coefficient was at the $95 \%$ confidence limit. The study checked the prediction performance between statistical models and ML methods. The daily interval trading dataset from April 30, 2013, to November 20, 2018, was used for the examination. GRU (RNN) as ML and The GARCH, ARIMA as traditional Statistical methods were also included. In case there are sufficient datasets to learn for RNN, they perform better than linear models such as GARCH while making predictions for the future by using past datasets. For this reason, in the study in question, the RNN model surpassed the GARCH model.

Furthermore, the same results were obtained in reference [11]. In the study, the researchers used the GARCH model, SMA, RNN (GRU). The GRU model performed better than SMA with the lowest Root Mean Square Error (RMSE) and mean absolute error (MAE) ratios.

Bayesian optimized RNN, LSTM, and ARIMA were used in another study. LSTM reached the maximal accuracy while the RNN reached the minimal RMSE. The ARIMA's predictions were poor for both. RNN yielded good results with a time lag of 50 days while LSTM used between 50 and 100 days for time lag where the best estimate was obtained with 100 days [12].

\subsection{Machine Learning-Machine Learning}

The least commonly used two or more ML algorithms for bitcoin price prediction were compared with each other. The dataset from January 1, 2012, to January 8, 2018, a 1-minute interval trading dataset was used. Theil-Sen Regression, Huber Regression, LSTM, GRU bitcoin price were predicted, and then GRU yielded the best accuracy result via Mean Squared Error (MSE) at 0.00002 and the R-Square (R2) was as high as $99.2 \%$ [13].

Based on reference [32], the researchers used three different ML models for bitcoin price prediction. These included LSTM, GRNN, and Nearest Neighbors In the study, the nonlinear structure of Bitcoin was investigated by using LSTM, Nearest Neighbors, and GRNN. LSTMs significantly surpassed the GRNN in terms of the RMSE. The researchers discovered that the generalized regression neural networks were not as successful as LSTM in finding patterns in addition to being time-consuming. 
Based on reference [28], the Bitcoin closing price was predicted by using ML methods, LR, L-SVM, and P-SVM. Bitcoin's closing price was predicted for MA and WMA filters by using daily closing price, highest price, and lowest price time series. P-SVM performed the best with 0.00075 MSE while LR performed the worst.

Based on reference [29], the researchers used a set of exogenous and endogenous variables to predict Bitcoin price. RNN and a GRU were proposed to predict Bitcoin price. RNN and LSTM are slow down than GRU, which can learn and train Bitcoin price fluctuations more adequately. The results of the study included RMSE of train-dataset NN 0.02 LSTM 0.010, GRU 0.010 while RMSE of test-dataset NN 00.31, LSTM 0.024, GRU 0.019 were also presented. Since LSTM and GRU are deep learning (DL) models, they perform better than traditional ML models.

Based on reference [30], the researchers used methods that covered ARIMA, LSTM, RNN, Gradient boosted trees (GBDT), Ensemble learning method, K-NN. Unlike other models, the K-NN model did not work rather effectively.

Of all the models used in the study, the best one is the ensemble learning method with a $92.4 \%$ accuracy rate and 0.002 RMSE value. The second place belongs to Gradient boosted trees with a $90 \%$ accuracy rate and 0.001 RMSE value. The GBDT is a ML technique prediction model in the form of a collection of poor estimation models, for gradient amplification, regression, and classification problems [31].

\subsection{Frequency Effect of Selected Time}

The effect of time on the dataset in bitcoin price prediction is via DL. The challenge of applying DL techniques is that algorithms require a large number of dataset samples to learn [14]-[16].

Based on reference [14], the researchers used time-series data with high-frequency returns on a 1-30 minutes scales. Since the dataset consisting of the price varies within 1-minute time intervals and it is highly volatile, the researchers preferred the data set with 30-minute intervals.

Based on reference [15], the period between May 6, 2014, and June 24, 2014, with Represent Bayesian regression algorithm was used to obtain the dataset of three different lengths, which covered X1 with a time-length of 30 minutes, X2 with a time-length of 60 minutes, and X3 with a time-length of 120 minutes. Then, interesting patterns were searched with the k-means algorithm in the datasets above. This is approximately, 89\% return in 50 days with X2. Since the result in the study [15] does not contain RMSE and MAE values, we cannot compare it with other studies.

Based on reference [16], the researchers found that the 10-minute dataset yielded better sensitivity and specificity compared to the 10-second dataset. With 10 minutes of the dataset, the researchers could see a clearer trend. This is because, in a smaller window, the price change can be predicted accurately.

However, the researchers recommended the 10-minute interval as a lower margin of profit, which was because the algorithm cannot send a trade in the 10-second window.

In this study, two main elements are used to obtain the bitcoin price prediction with the lowest RMSE value. Considering the previous articles, it was observed that the best results were more frequently spaced, that is, they covered a minute, a second, 10-minute, 10-second datasets.

Based on reference [16], the researchers used two separate time interval datasets and discovered that the 10-minute dataset yielded better sensitivity and specificity ratios compared to the 10-second dataset. The dataset consists of 30, 60, 120-hour bitcoin prices.

Based on reference [15], the researchers constructed a time series with time 10-second intervals between February 2014 and July 2014. Unlike reference [16], the time interval used was quite large and the researchers obtained more than 10 million cross-correlations. The researchers used different threshold " $\mathrm{t}$ " to simulate the trading strategy and see how the performance of the strategy changed. The researchers used two models for time-length as 30, 60, 120 minutes and 180, 360, 720 minutes. Concretely, as the threshold increase, the number of trades decreased and the average holding time increased. At the same 
time, the average profit per trade increased. This resulted in almost a two-fold increase in investment in 50 days.

The average of the minute and second dataset during the day yielded good results in studies that conducted daily average value estimates.

For example, based on reference [13] ,by using 1-minute interval trading dataset on Theil-Sen Regression, LSTM, Huber Regression, and GRU by predicting bitcoin prices, GRU yielded the best accuracy result via MSE at 0.00002. However, we are interested in LSTM and its MSE result is 0.000431 . Both results are a prediction of the average price of the day.

Furthermore, since there were more gaps and losses in the second set of datasets in the previous articles, a dataset of 1, 30, 60,120, 180 minutes was created and used on the network. It can be stated that by looking at studies [13], [15], [16], when conducting bitcoin price predictions, the use of hour, minute, second datasets, instead of a daily dataset, provided better results. This was because the dataset was enlarged and the frequency was increased. Therefore, both minute and daily prices were used for the comparisons.

\subsection{Effect of Social Media and Web Search Engine}

Whether bitcoin's price variation was related to the volume of tweets or Web Search engine results were investigated and it was discovered that there was a significant relationship between Google Trends data and tweets, which was a positive mood for bitcoin's price. The result confirmed that the quantities of exchanged tweets could predict fluctuations in Bitcoin price. Moreover, in this study, the effect of the comparisons between the positive and negative tweet trends regarding the Bitcoin price was also observed as a reason for Bitcoin's thriving popularity while a boost in search volumes resulted in an upward trend in tweets [17]. Furthermore, numerous studies, such as [18], [19], [20], were conducted on this subject. Although all of them reported a little impact on bitcoin price prediction, researchers continue to examine the relationship between bitcoin and Twitter, Google Trends.

\subsection{Causalit}

Generally, the relationship between bitcoin, gold, and dollar was evaluated by statistical methods. Two studies [21], [22] used the GARCH model. This is because Bitcoin, gold, and dollar react to similar variables in the GARCH model [22]. However, in the study [21], it was stated that Bitcoin was rather different from gold and fiat currencies. ARDL and NARDL models were used in the study [23] and it was discovered that there was no connection between bitcoin and gold price in the short and long terms while there was no linear and quantile relationship. In the study [24], the same model, ARDL, and same the result indicated that gold price had no significant impact on Bitcoin price.

Based on reference [25], the researchers found that while the market is normal, the volume can be used to predict returns.However, when the market shows fluctuating performance, volume remains irrelevant because only the historical price was significant for predicting future returns. We can see the effect of dollar and gold on performance by investigating the studies that used the deep neural network, which is believed to be the best method to estimate the bitcoin price such as study [26]. The effect of gold price on bitcoin price prediction was examined using CNN, LSTM, and GRU models. LSTM results in the least RMSE value for bitcoin price prediction. However, when the gold price is included in the network as the parameter for bitcoin price prediction, LSTM, CNN, GRU models do not result in a positive correlation between the gold price and bitcoin price.

Based on reference [27], considering the unique characteristics of Bitcoin and its trivial relations with the fluctuations in the macroeconomics, it can be stated that the Bitcoin market has a weak relationship with many assets.

Additionally, pieces of evidence of the link between markets in the short, medium, and long term were demonstrated, which indicated that cryptocurrencies were relatively isolated from market shocks and separated from popular financial assets based on findings of reference [36]. 


\subsection{Optimization of Hyperparameters}

Setting hyper-parameters has always been a problem. The most important reason is that hyperparameters are datasets and they are model specific. Hyperparameters that provide the best results for a dataset or model do not have the same effect when the dataset or model changes. Classic hyper-parameters in neural networks include network layers, weight initialization, , number of neurons in each layer, and learning rate [37]. They are evaluated only in terms of the learning rate.

Based on reference [38], a wide range of parameters to determine the effects of learning rates include batch sizes, momentum, different non-linearities, and peepholes.

In the next report; when conducting bitcoin price prediction with LSTM, hyperparameters optimization will be used manually and the activation function, optimizer, batch size, learning speed, periods, network layers, number of neurons in each layer will be evaluated.

Hyperparameter optimization for bitcoin price prediction was chosen for the next report because the manual or automatic hyperparameters optimization has never been conducted before.

\section{Materials and Methods}

Previous studies on Bitcoin price prediction are grouped under certain headings. These groups include (1) Statistical Methods, (2) Machine Learning-Statistical Methods, (3) Machine Learning-Machine Learning, (4) Effect of Selected Time, (5) Effect of tweets, and Web Search Media, (6) Causality, (7) Optimization of Hyperparameters.

The literature review was conducted for each of the topics mentioned above and the results were compared and summarized in the "Results \& Discussion" section. Starting with the highest number of stars, scoring was conducted by giving the algorithm the best result of the methods that were compared according to the error rates (MAE, MSE, MAPE, and RMSE) or the criteria in the study.

In this report, it was aimed to determine whether the bitcoin market had a relationship with price fluctuations in gold, crude oil, natural gas, the dollar-euro parity, ethereum, and other cryptocurrencies. We wanted to figure out the causality relationship between bitcoin and gold, crude oil, natural gas, ethereum, the dollar-euro parity based on the seven different models, which included MLP, SVM, Generalized Regression Neural Network (GRNN), RNN, LSTM, GRU, Convolutional Neural Network (CNN) or statistical methods. Then, the best model was determined by using the error function, which includes RMSE and MAE. For causality relationship at any instance, the past samples of data were placed in the network input while the subsequent values were predicted at its output. Six different datasets will be used with seven different models, which cover MLP, SVM, GRNN, RNN, LSTM, GRU, and CNN.

\section{Results and Discussion}

This report is conducted for the analysis and comparison of prediction bitcoin price by using ML and Statistical Methods. For bitcoin price prediction, the DL methods in time series forecasting are expected to be better than the traditional ML models and statistical models.

The previous experimental studies on forecasting cryptocurrency prices provided evidence regarding the fact that this is true. Considering the number of layers in ANNs, the combination of nodes between multiple layers is known to increase the ANN representation. Additionally, several recent studies proved that the efficiency of ANN representation increased exponentially in terms of certain function classes compared to the increase in the number of layers [39]. However, generally, it was observed that the increase in the layers does not increase the yield after a certain point and vice versa [40].

Based on reference [5], the researchers compared input selection in ARIMA, VAR while using SVR and MLP networks. This process, also called Hybrid Model Experiments, demonstrated that the SVR method outperformed the MLP networks for each input selection algorithm. 
When the models are compared, the input we selected is vital and it affects the results [5]. Therefore, when the dataset is sparse or when there is an insufficient dataset to train the network, the result of ML and statistical methods may be close, which may yield better results even by using statistical methods. This does not mean that statistical methods are better than ML. In recent days, there is a growing consensus that analyzing particularly complex patterns of DL neural networks and predictions using these patterns are more effective than traditional topologies [32].

Below, we compared the results of ML methods with conventional statistical methods while occasionally covering hybrid structures that use both.

Accordingly, the following results were presented.

* ARIMA yields the best results among the statistical methods [7].

* ARIMA remains weak compared to RNN, LSTM [12], [30] and SVM, MLP [6], [11].

*As a result, it was proved that ML methods produced better results compared to statistical methods.

*Based on references [6], [11] and [12], when the traditional ML models, such as SVM, MLP, RNN, were compared, we can state that MLP yields the best results.

*GRU yields the best results among the ML methods. The researchers made the best estimate with an error value of 0.00002 MSE by using the Closed, Open, High, and Low prices to estimate the intraday weighted price in reference [13].

* As a result, it was proved that DL methods produced better results compared to traditional ML.

* In the comparison of ML and statistical methods, ML algorithms yield better results.

* In the comparison of Traditional ML and DL models, DL algorithms yield better results.

Table 1 Comparison of ML and statistical methods

\begin{tabular}{|c|c|c|c|c|c|c|c|c|c|c|c|c|}
\hline Accuracy & ARIMA & AR & MA & $\begin{array}{l}\text { Theil-Sen } \\
\text { Regression }\end{array}$ & $\begin{array}{c}\text { Huber } \\
\text { Regression }\end{array}$ & $\begin{array}{c}\text { Random } \\
\text { Forest }\end{array}$ & MLP & SVM & RNN & LSTM & GRU & GRNN \\
\hline [12], [30] & $*$ & & & & & & & & $* *$ & $* * *$ & & \\
\hline [13] & & & & $* *$ & $* * *$ & & & & & $*$ & $\begin{array}{l}* * * \\
*\end{array}$ & \\
\hline$[16]$ & & & & & & $*$ & & $* *$ & & & & \\
\hline [35] & & & & & & & & $*$ & & $* *$ & & \\
\hline [41] & & & & & & & $* *$ & & * & $* * *$ & & \\
\hline [7] & $* * *$ & ** & * & & & & & & & & & \\
\hline $\begin{array}{l}6],[11], \\
{[12]}\end{array}$ & $*$ & & & & & & $\begin{array}{l}* * \\
*\end{array}$ & $* *$ & & & & \\
\hline$[29]$ & & & & & & & & & $*$ & $* *$ & $* * *$ & \\
\hline$[32]$ & & & & & & & & & & $* *$ & & $*$ \\
\hline
\end{tabular}

Below, we compared the results of studies on gold, crude oil, natural gas, ethereum, the dollar-euro parity, or other commodities that may have a causality relationship with bitcoin.

Accordingly, the following results were presented.

*Based on references [22], [23], [25] and [26], we can say that there is no correlation between gold, dollar and bitcoin price and in reference [21], it was stated that Bitcoin was rather different from gold and fiat currencies.

Based on reference [36], the researchers stated that cryptocurrencies were relatively isolated from market shocks and separated from popular financial assets.

However, based on reference [24], the gold price had no significant impact on Bitcoin price and based on reference [27], it can be stated that the Bitcoin market is in a weak relationship with many assets. 


\section{Conclusion and Future Work}

In terms of Covid-19's effect on Bitcoin price, after rising from \$ 7,200 on January 20, 2020 to \$10,000 between January and February, the price on January 1, 2020 went down with Covid-19 at the end of February. On March 13, 2020, with a harsh decrease to \$5,000 levels occurred, which coincided with the date when many countries observed Covid-19 cases, such as Covid-19 cases in Turkey and Italy. Furthermore, this state could be related to the effect of the increasing number of deaths. Following the sharp drop in Bitcoin, an upward trend with fluctuation from March 20, 2020, to June was observed. Then, it attracted attention and experienced increases, which turned the negative effect of Covid-19 into a positive effect and surpassed the \$10,000 band. Even in a global pandemic phase, bitcoin has remained a preference for investors. This shows that bitcoin will continue to be pursued with great interest just as studies on its price prediction as it continues to maintain its appeal for investors.

Thanks to a detailed literature review on price prediction with bitcoin, it was observed that there was no study on automatic or manual hyperparametric optimization in bitcoin price estimation.

\section{References}

[1] M. Rahouti, K. Xiong and N. Ghani, "Bitcoin Concepts, Threats, and Machine-Learning Security Solutions," in IEEE Access, vol 6, pp. 67189 - 67205, 9 November 2018.

[2] S. Abboushi, "Global Virtual Currency - Brief Overview.19(6), 10-118.," The Journal of Applied Business and Economics, vol 19, no. 6, 102017

[3] O. D. Juarez and H. E. Manzanilla , "Forecasting Bitcoin Pricing with Hybrid Models A Review of The Literature," International Journal of Advanced Engineering Research and Science (IJAERS), vol 6, no. 9, pp. 161-164, Sept 2019.

[4] H. Garrick and M. Rauchs, "Global cryptocurrency benchmarking study," Cambridge Centre for Alternative Finance, 2017.

[5] H. Ince and T. B. Trafalis, "A Hybrid Model for Exchange Rate Prediction," Decision Support Systems, vol 42, p. 1054-1062, 2006.

[6] I. N. Indera, I. M. Yassin, A. Zabidi and Z. I. Rizman, "Non-Linear Autoregressive with Exogeneous Input (NARX) Bitcoin Price Prediction Model Using PSO-Optimized Parameters Optimized Parameters," Journal of fundamental and applied sciences, vol 9, no. 3S, p. 791, 10 September 2017

[7] S. Roy , S. Nanjiba and A. Chakrabarty, "Bitcoin Price Forecasting Using Time Series Analysis," ICCIT, Dhaka, Bangladesh, 2018

[8] A.Zeba; F.Jinan ; S.Ahmer ; A.M. Anwer, "Bitcoin Price Prediction using ARIMA Model," Canada, 2020.

[9] P. Katsiampa, "Volatility Estimation for Bitcoin: A Comparison of GARCH Models," Economics Letters, vol 158, pp. 3-6, 2017. 
[10] N. Mangla, A. Bhat, G. Avabratha and N. Bhat, "Bitcoin Price Prediction Using Machine Learning," International Joutnal of Information And Computing Science, vol 6, no. 5, pp. 318320, May 2019.

[11] S. Ze, W. Qing and L. J. David, "Model, Bitcoin Return Volatility Forecasting: A Comparative Study of GARCH Model and Machine Learning,"Agricultural \& Applied Economics Association, Atlanta, GA, 2019.

[12] M. Sean, R. Jason and C. Simon, "Predicting the Price of Bitcoin Using Machine Learning," 26th Euromicro International Conference on Parallel, Distributed, and Network-Based Processing, 2018.

[13] P. Thearasak and N. Thanisa, "Machine Learning Models Comparison for Bitcoin Price Prediction," 2018 10th International Conference on Information Technology and Electrical Engineering (ICITEE), pp. 506-511, 2018.

[14] T. Shintate and . L. Pichl, "Trend Prediction Classification for High Frequency Bitcoin Time Series with Deep Learning," Risk and Financial Management, 2019.

[15] S. Devavrat and Z. Kang , "Bayesian regression and Bitcoin," Fifty-second Annual Allerton Conference, Monticello, IL, USA, 2014.

[16] I. Madan, S. Saluja and A. Zhao,"Automated Bitcoin Trading via Machine Learning Algorithms," Google Scholar, 2014.

[17] M. Martina , L. Ilaria and M. Michele , "Bitcoin Spread Prediction Using Social And Web Search Media," UMAP Workshops, 2015, 2015

[18] J. C. Kaminski and M. M. Lab, "Nowcasting the Bitcoin Market with Twitter Signals," Cornell University, Jan 2016.

[19] D. Garcia, C. J. Tessone, P. Mavrodiev and N. Perony, "Feedback Cycles Between SocioEconomic Signals in the Bitcoin Economy," The Journal of The Royal Society, Oct 2014.

[20] D. Shen, A. Urquhart and P. Wanga, "Does Twitter Predict Bitcoin?," Economics Letters, vol 174, p. 118-122, 2019.

[21] D. G. Baur, T. Dimpfl and K. Kuck, "Bitcoin, Gold and the US Dollar - A Replication and Extension," Finance Research Letters, vol 25, pp. 103-110, 2018.

[22] A. H. Dyhrberg, "Bitcoin, Gold and The Dollar -A GARCH Volatility Analysis," Finance Research Letters, vol 16, pp. 85-92, 2016.

[23] E. Bouri, R. Gupta, A. Lahiani and M. Shahbaz, "Testing for Asymmetric Nonlinear Short- and Long-Run Relationships Between Bitcoin, Aggregate Commodity and Gold Prices," Elsevier, vol 57, p. 224-235, 2018. 
[24] J. Bouoiyour and R. Selmi, "What Does Bitcoin Look Like?," Annals of Economics and Finance, vol 16, no. 2, pp. 449-492, 2015.

[25] M. Balcilar, E. Bouri, R. Gupta and D. Roubaud, "Can Volume Predict Bitcoin Returns and Volatility? A Quantiles-Based Approach.," Economic Modelling, 2017.

[26] A. Aggarwal, I. Gupta, N. Garg and A. Goel, "Deep Learning Approach to Determine the Impact of Socio Economic Factors on Bitcoin Price Prediction," Twelfth International Conference on Contemporary Computing (IC3), India, 2019.

[27] M. Polasik, A. I. Piotrowska, T. P. Wisniewski, R. Kotkowski and G. Lightfoot, "Price Fluctuations and the Use of Bitcoin: An Empirical Inquiry," International Journal of Electronic Commerce, vol 20, no. 1, pp. 9-49, 2015.

[28] S. Karasu, A. Altan, Z. Saraç and R. Hacioğlu, "Prediction of Bitcoin Prices with Machine Learning Methods Using Time Series Data," 2018 26th Signal Processing and Communications Applications Conference (SIU), Izmir, Turkey, May 2018.

[29] A. Dutta, S. Kumar and M. Basu, "A Gated Recurrent Unit Approach to Bitcoin Price Prediction," Journal of Risk and Financial Management, vol 13, no. 2, Feb 2020

[30] R. Chowdhury, M. A. Rahman, M. S. Rahman and M. Mahdy, "An approach to predict and forecast the price of constituents and index of cryptocurrency using machine learning,» Physica A: Statistical Mechanics and its Applications, vol 551, April 2020.

[31] J. H. Friedman, "Stochastic Gradient Boosting," Computational Statistics \& Data Analysis, vol 38, pp. 367-378, 2002.

[32] S. Lahmiri and S. Bekiros, "Cryptocurrency Forecasting with Deep Learning Chaotic Neural Networks," Chaos,Solitons and Fractals, vol 118, pp. 35-40, 2019.

[33] F. A. Narudin, A. Feizollah, N. B. Anuar and A. Gani, "Evaluation of Machine Learning Classifiers for Mobile Malware Detection," Soft Computing, no. 20, p. 343-357, 2014.

[34] F. Long, K. Zhou and W. Ou, "Sentiment Analysis of Text Based on Bidirectional LSTM With Multi-Head Attention," IEEE Access, vol 7, pp. 141960 - 141969, Sep 2019.

[35] C. Zheshi, L. Chunhong and S. Wenjun, "Bitcoin Price Prediction Using Machine Learning: An Approach to Sample Dimension Engineering," Journal of Computational and Applied, vol 365, July 2019.

[36] S. Corbet, A. Meegan, C. Larkin, B. Lucey and L. Yarovaya, "Exploring the Dynamic Relationships Between CryptoCurrencies and Other Financial Assets," Economics Letters, vol 165, pp. 28-34, 2018.

[37] C. Yu, X. Qi, H. Ma, X. He, C. Wang and Y. Zhao, "LLR: Learning Learning Rates by LSTM for Training neural Networks," Neurocomputing, pp. 22-45, Feb 2020. 
[38] T. M. Breuel, "Benchmarking of LSTM Networks," Cornell University, 2015.

[39] Y. Bengio, A. Courville and P. Vincent, "Representation Learning a Review and Perspectives," IEEE Transactions on Pattern Analysis and Machine Intelligence, vol 35, no. 8, August 2013.

[40] M. Nakano, A. Takahashi and S. Takahashi, "Bitcoin Technical Trading with Artificial Neural Network," Physica A, vol 510, p. 587-609, 2018.

[41] B. Spilak, "Deep Neural Networks For Cryptocurrencies Price Prediction," Thesis Master in Humboldt University , Berlin, 2018. 